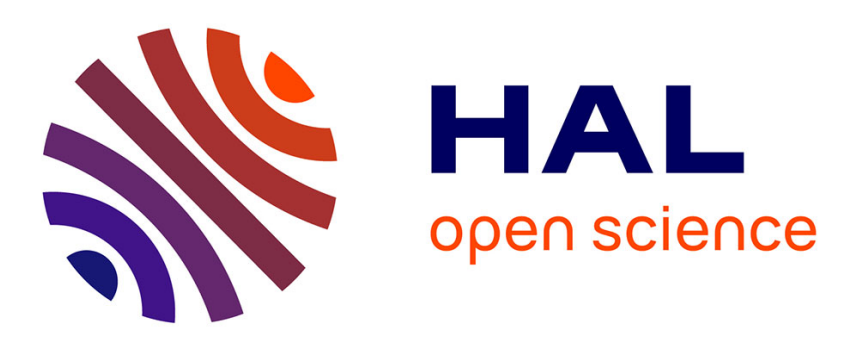

\title{
COSMIC: A Cooperative MAC Protocol for WSN with Minimal Control Messages
}

\author{
Ahmed Ben Nacef, Sidi-Mohammed Senouci, Yacine Ghamri-Doudane, \\ André-Luc Beylot
}

\section{- To cite this version:}

Ahmed Ben Nacef, Sidi-Mohammed Senouci, Yacine Ghamri-Doudane, André-Luc Beylot. COSMIC: A Cooperative MAC Protocol for WSN with Minimal Control Messages. 4th IFIP International Conference on New Technologies, Mobility and Security (NTMS'11), Feb 2011, Paris, France. pp.1-5, 10.1109/NTMS.2011.5721060 . hal-00794952

\section{HAL Id: hal-00794952 \\ https://hal.science/hal-00794952}

Submitted on 7 Mar 2013

HAL is a multi-disciplinary open access archive for the deposit and dissemination of scientific research documents, whether they are published or not. The documents may come from teaching and research institutions in France or abroad, or from public or private research centers.
L'archive ouverte pluridisciplinaire HAL, est destinée au dépôt et à la diffusion de documents scientifiques de niveau recherche, publiés ou non, émanant des établissements d'enseignement et de recherche français ou étrangers, des laboratoires publics ou privés.

\section{(ㄷ)(1) $\$$}

Distributed under a Creative Commons Attribution - NonCommercial| 4.0 International 


\title{
COSMIC: A Cooperative MAC Protocol for WSN with Minimal Control Messages
}

\author{
Ahmed Ben Nacef*, Sidi-Mohamed Senoucill, Yacine Ghamri-Doudane ${ }^{\S * *}$ and André-Luc Beylot \\ *2, avenue Pierre Marzin 22307 Lannion Cedex, France \\ Email: ahmed.bennacef@ orange-ftgroup.com \\ "Université de Bourgogne, ISAT Nevers, 49 Rue Mademoiselle Bourgeois, 58000 Nevers, France \\ Email: Sidi-Mohammed.Senouci@u-bourgogne.fr \\ ${ }^{\S}$ ENSIIE, 1 Square de la résistance, 91025 Evry CEDEX, France \\ Email: ghamri@ensiie.fr \\ IENSEEIHT, Laboratoire IRIT, 2 rue C. Camichel, BP 7122, 31071 Toulouse Cedex 7, France \\ Email: beylot@enseeiht.fr \\ **LIGM - Université de Paris-Est Marne-la-Valle, 75420 Champs sur Marne, France
}

\begin{abstract}
Over the last decade cooperative communication in wireless sensor networks (WSN) received much attention. A lot of works have been done to propose a MAC layer that supports cooperative relaying. The majority of these works tried to adapt the IEEE 802.11 MAC protocol to sensor networks. The adapted protocols use a lot of overhead (such as the use of RTS/CTS as well as other messages used to allow cooperation) that consumes energy. In this paper we propose a CSMA/CA based MAC protocol that supports cooperative communication with a minimum overhead: COSMIC (A Cooperative MAC Protocol for WSN with Minimal Control Messages). Relay selection in this new protocol is performed using both the channel state information (CSI) and the remaining energy. Simulation results show that COSMIC is able to increase the network lifetime by $25 \%$
\end{abstract}

Index Terms-Cooperative relaying, relay selection, energy efficiency, wireless sensor networks.

\section{INTRODUCTION}

Low Power and Lossy Networks such as Wireless Sensor Networks (WSN) attracted much attention during the last years. The restricted resources (energy, memory, and processor) and the random quality of the channel characterizing such networks rise up multiple challenges. Several techniques and protocols were proposed to meet this constraints [1], [2]. One of the most interesting alternatives to enhance the channel capacity and to reduce the energy consumption is to use cooperative relaying [1]. The direct link between a source $\mathrm{S}$ and a destination $\mathrm{D}$ can be affected by fading. At the same time, S may have some neighbors that experience better channel conditions to the destination and that over-hear all the packets sent by S. We can exploit this spatial diversity and retransmit the packet from one of the neighbors instead of retransmitting it from the source.

Let us take the case of Figure 1 as an example. When the source $\mathrm{S}$ sends a packet to the destination $\mathrm{D}$, it is overheard by the neighbors $\mathrm{N} 1$ and $\mathrm{N} 2$ of $\mathrm{S}$ and $\mathrm{D}$. The conditions of the channel from $\mathrm{S}$ to $(\mathrm{N} 1, \mathrm{~N} 2)$ and from $(\mathrm{N} 1, \mathrm{~N} 2)$ to $\mathrm{D}$ are not the same as the direct channel from $\mathrm{S}$ to $\mathrm{D}$. In addition if the direct channel is bad the packet may arrive corrupted to $\mathrm{D}$. In this case, the neighbor with a better channel to $\mathrm{D}$ and that correctly received the packet, retransmits it instead of $\mathrm{S}$. This retransmission on a better path will avoid the source retransmitting the packet several times on a poor channel.

As shown in Figure 1 cooperative relaying is composed of three phases: direct transmission, relay selection and cooperative transmission. The source $\mathrm{S}$ sends directly a message to the destination $\mathrm{D}$, we call this step by direct transmission. A transmission from a neighbor to the destination is called cooperative transmission. Relay selection is the action of choosing the neighbor(s) able to relay correctly the packet to $\mathrm{D}$ (hereafter we call this special neighbor by relay). Two main relaying strategies exist. The first consists in employing several neighbors to relay the packet at the same time, which becomes possible by using Space Time Coding (STC) [3]. The second consists in using only one relay at a time [4], which can outperform the use of multiple relays [5].

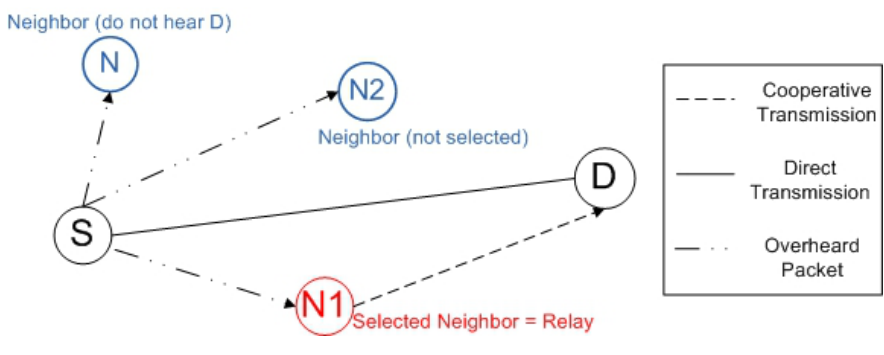

Fig. 1. Cooperative Relaying

The remainder of this paper is organized as follows: in section 2 we discuss related works, in Section 3 we present the details of our protocol. Section 4 presents and discusses the performance evaluation results. Finally, we summarize the main results and introduce our future work in Section 5.

\section{RELATED WORKS}

Multiple research efforts were proposed to include cooperative relaying in the network layer [6], [7] or in the MAC 
layer [4], [8]-[13]. In this paper we focus only on the efforts made at the MAC layer. The majority of these cooperative MAC protocols use the Channel State Information (CSI) to decide which neighbor to elect as relay [4], [11]-[13]. The source and the destination of the packet exchange Ready-ToSend(RTS)/Clear-To-Send (CTS) messages in order to make a channel reservation. The neighbors hear these RTS/CTS, deduce from them the CSI of the channels to the destination and the source and based on that they compute a back-off timer. Subsequently they enter into a contention period to gain the channel. The timer of the neighbor eligible to become the relay (i.e. the one with the best CSI) expires first and it applies for the relaying.

In addition to CSI, other protocols propose to consider energy in relay selection [10]. The authors propose to combine the CSI of the channel to the remaining energy of the node to elect the relay. Energy conservation was the subject of other considerable research efforts. Z-MAC [14] and B-MAC [15] proposed the use of Low Power Listening to reduce the activity time of the nodes. These type of techniques is out of the scope of our paper.

The majority of these protocols was conceived for 802.11 networks or adapted from 802.11 to WSN. Therefore they do not completely meet the constraints of WSN (especially with regard to energy and restricted channel capacity). The objective of this paper is to present COSMIC; our Carrier Sense Multiple Access/Collision Avoidance (CSMA/CA) based cooperative MAC protocol with a distributed relay selection algorithm. The selection decision is based on both channel state and remaining battery and it uses only one control packet for relay selection.

\section{The COSMIC PROTOCOL}

\section{A. Motivation}

The majority of cooperative MAC protocols designed for WSN were inspired from the 802.11 MAC layer. Therefore they use the RTS/CTS sequence and other control messages for relay selection and cooperative transmission. Besides WSNs are much more energy constrained (often use non-rechargeable batteries) and they dispose of a limited data rate. Furthermore the RTS/CTS of 802.11 was conceived to protect the transmission of large data packet while the maximum size of a WSN packet is of 127 bytes. Therefore, RTS/CTS mechanism is not really necessary for wireless sensor networks.

The relay selection mechanism is usually based on the CSI of the source-to-relay, source-to-destination and relayto-destination channels, without considering a second critical parameter which is the residual energy of the nodes.

Furthermore, several works perform a proactive relay selection. They select the relay before data transmission and use a probabilistic decision. However, this probabilistic decision is not needed if we make a reactive relay selection, i.e. after the data transmission. The process become deterministic as each node knows if it has correctly received the data packet and can definitely decide if it can be the relay or not.

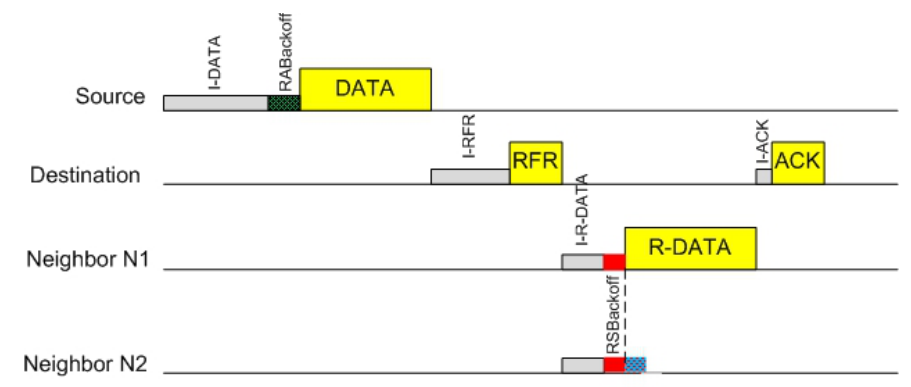

Fig. 2. COSMIC

To sum up, we can say that we strongly believe that more energy and channel capacity can be saved if we reduce the number of control packets and if we perform a reactive relay selection.

\section{B. Protocol Description}

In this section we describe the details of our protocol: the queuing strategy, the inter-frame spacing and the different backoffs. By designing COSMIC we aim to minimize the use of the radio by reducing the number of exchanged control packets. More precisely we use timers instead of control messages. Hereafter we will use the following notations: DATA which is the data packet, Request-For-Relay (RFR), Acknowledgment (ACK) and R-DATA which is the relayed copy of the data packet.

Let us take the example depicted in Figure 1. We suppose that we have a one-hop communication between a source $S$ and a Destination D in presence of two common neighbors N1 and N2. When S has a DATA packet to send, it has to wait and the inter-frame DATA (I-DATA) period plus a random backoff period (RABackoff), after which it sends the packet to D if the channel is free. D receives the packet and the neighbors overhear it. Only the neighbors that are common to $\mathrm{S}$ and D keep the over-heard packet (N1 and N2 in our case). The other neighboring nodes drop the packet silently. If $\mathrm{D}$ is able to decode the packet, it sends an acknowledgment (ACK) to S. In this case, no relaying is needed. The potential relays drop the over-heard packet. Otherwise, if D was not able to decode the DATA packet, a cooperative relaying is engaged.

Figure 2 describes the sequence of messages used to send one packet from $\mathrm{S}$ to $\mathrm{D}$ with cooperative relaying. $\mathrm{D}$ sends an RFR, after an RFR Inter-frame spacing (I-RFR), to express its need for a relayed packet. The neighbors receive the RFR and deduce the CSI of the channel to D then compares it with a predefined threshold TH. If the measured CSI is above this threshold, the neighbor is considered as a potential relay, otherwise the neighbor deduces that it cannot enhance the direct transmission and retires silently. At this step, in our example, all the remaining neighbors are able to relay the data packet to D (i.e. N1 and N2 in Figure 1). These neighbors will enter a contention period and compete for packet relaying. In order to become the relay, each potential relay computes an RSBackoff using equation (3). 


$$
\begin{aligned}
& R S B \text { ackoff }=\frac{1}{\alpha \times N(C S I)+\beta \times N(R E)} \\
& N(C S I)=\frac{C S I}{C S I_{M A X}} \\
& N(R E)=\quad \frac{R E}{\text { InitialEnergy }}
\end{aligned}
$$

Where $\mathrm{N}(\mathrm{CSI})$ is the normalized value of instantaneous CSI of the R-D channel deduced from the signal of the received packets. $\mathrm{N}(\mathrm{RE})$ is the normalized value of the remaining energy of the relay R. $\alpha$ and $\beta$ are the correspondent weights of the $\mathrm{N}(\mathrm{CSI})$ and $\mathrm{N}(\mathrm{RE})$ respectively. They define the decision strategy of the relay. InitialEnergy and $C S I_{M A X}$ are respectively the initial available battery of the neighbor and the maximum possible value of the CSI.

In our example the RSBackoff of $\mathrm{N} 1$ expires first and relays afterward the packet. N2 hears this transmission, cancels its scheduled one and drops the packet. At the destination side, if the packet is decoded, D sends an ACK to S, otherwise, it remains silent. Once the re-transmission timer expires without receiving an ACK, the source deduces that a retransmission is required.

\section{Packet Priority and NAV computing}

We define in this section the priority order between the packets, in addition to the Network Allocation Vector (NAV) that will reduce the collision probability and determine some periods of inactivity.

In our approach, we suppose that a cooperative communication has to be proceeded before any other data packet communication, since we consider that relaying a packet accomplishes an unfinished communication between the source and the destination. Therefore, we assign a higher priority to potential relays. In order to ensure this priority, we define a number of inter-frames. When sending DATA, a node has to wait for Inter-frame DATA (I-DATA) plus a Random backoff (RABackoff). We assign to I-DATA the highest value among the other inter-frames to make it the lowest priority to access the medium. We have now to define the priority order between the RFR and R-DATA packets. Let us suppose that RFR has higher priority compared to R-DATA. In this case, several relaying processes can be initiated at the same time and the same neighborhood, then this can engender an additional delay on the relaying of the packet by the potential relay and can engender a retransmission by the source. For these reasons, we opt to assign higher priority to R-DATA. Therefore we assign to the interframe of the R-DATA, smaller value than I-RFR. The packet priority order is thus as follows: DATA $<$ RFR $<$ R-DATA $<$ ACK and the corresponding inter-frame spacing order is as follows:

\section{I-ACK $<$ I-R-DATA $<$ I-RFR $<$ I-DATA}

A second solution to reduce the number of collisions is the use of the Network Allocation Vector (NAV). Each time a node can preview that traffic will be sent, it sets its NAV.

In our case, a node can forecast the traffic only when it receives an RFR. Two cases are possible: the packet can be relayed by a neighbor or not. In the first case, the neighbors will wait for I-R-DATA and RSBackoff then relay the packet. At the reception of the packet, the destination will respond with an ACK. If we suppose that the propagation delay is negligible, the time RLY-D required for this communication is as follows:

$$
\text { RLY-D = I-R-DATA + RS-Backoff + Max }\left(\frac{\text { size }(R-D A T A)}{\text { DataRate }}\right)
$$
$+\mathrm{I}-\mathrm{ACK}$

In the other hand, if the packet cannot be relayed, a timer will expire at the source and destination side. The waiting time is as follows :

$$
\text { RLY-D = I-R-DATA + Max(RSBackoff })+\epsilon
$$

Where $\epsilon$ is a predefined constant. In order to reduce collisions and unnecessary channel sensing, the neighbors that are not involved in the relay process will behave as follows: When hearing an RFR, they remain idle until the expiration of the No-RLY-D timer, then they sense the channel. If an activity is heard, they return to idle state until the end of RLY-D. If not, they can send their own packets.

\section{Performance Evaluation}

\section{A. Simulation Environment}

We implemented COSMIC on Opnet 15.0 simulator. The simulated sensors are equipped with a unique antenna and are not able to send and receive at the same time. They all use the same channel in the $2.4 \mathrm{GHz}$ band. Without loss of generality, we suppose that the sensor nodes can be in one of the following three states: transmitting, receiving or idle. Each sensor is equipped with an AA battery. The energy consumption in each state is of a Mica2 sensor node [17]: $27 \mathrm{~mA}$ for transmitting, $10 \mathrm{~mA}$ for receiving and $20 \mu \mathrm{A}$ for idle mode. The source $S$ sends a data packet to $D$ each minute. We used a 1016-bits data packet. RFR and ACK packets sizes are 88 bits. The data rate is fixed to $250 \mathrm{~Kb} / \mathrm{s}$. The topology of the network is fixed and uniform. In addition, we suppose that all the channels are symmetric, and that the radio range of each node is about $30 \mathrm{~m}$.

The route from the source $\mathrm{S}$ to the destination $\mathrm{D}$ is fixed during the simulation and contains an intermediate node I. Furthermore we uniformly distribute the neighbors around S, I and $\mathrm{D}$ in a square with a side length of $100 \mathrm{~m}$. At the beginning of the simulation, all the nodes have the same amount of energy and no other traffic is disturbing the packets from $\mathrm{S}$ to D. We vary the number of nodes from 10 to 100 .

The potential relays are the nodes on the radio range of the sender and the receiver at the same time. A potential relay decides to compete for relaying a data packet if the CSI measured from the RFR is higher than the threshold TH. The RSBackoff is computed using this CSI and the remaining percentage of energy. The signal $x(t)$ transmitted by a node over the channel experiences attenuation described by a fading coefficient $\mathrm{h}$, which is, random, but constant during the transmission of one data packet, as well as additive white noise $n(t)$. Therefore the signal received by $D$ at an instant $t$ is $y(t)=h \cdot x(t)+n(t)$. During the simulations we vary $n(t)$, 
randomly and for each packet, so that we simulate random channel conditions for every packet.

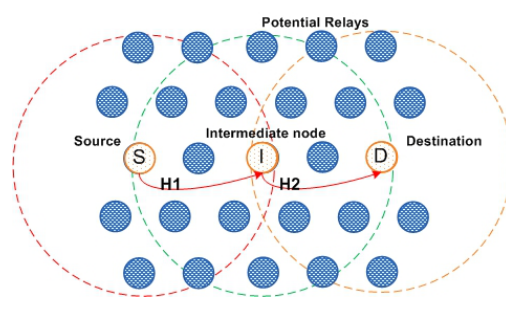

Fig. 3. Simulated Network

\section{B. Simulation Results}

In order to compare the performance of COSMIC to those of the CSMA/CA, we simulate the network described in Fig. 3 . We used the following performance metrics : the network lifetime, the delivery ratio and finally the outage ratio of the two hops S-I and I-D.

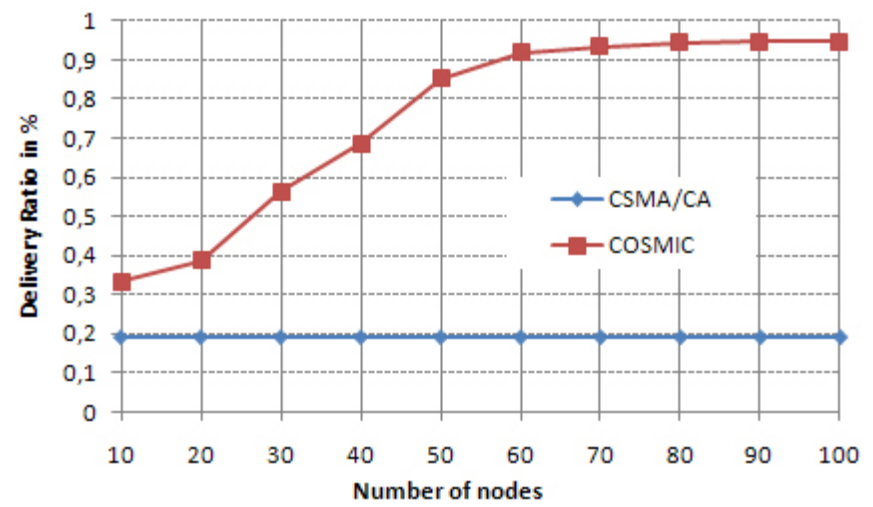

Fig. 4. Delivery ratio Vs Number of nodes

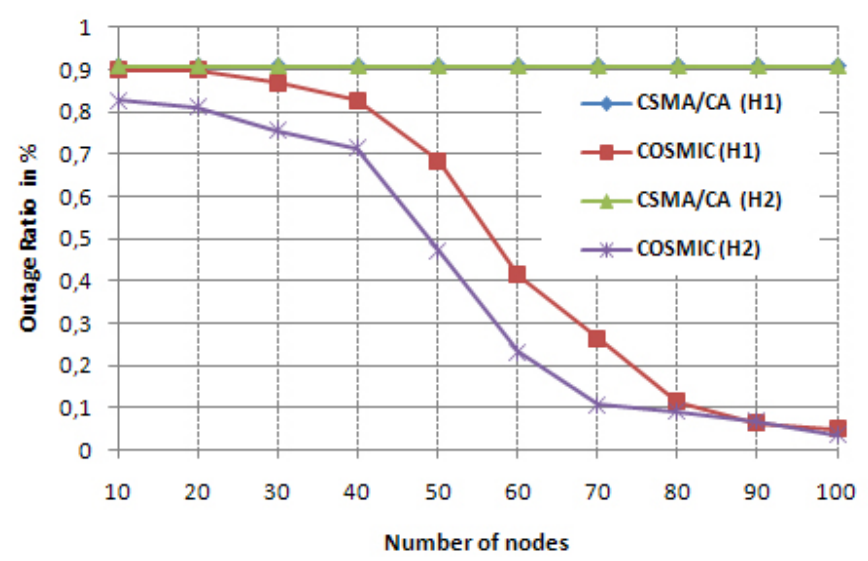

Fig. 5. Outage Ratio Vs Number of Nodes

In Fig. 4 Delivery Ratio for different node density are depicted. It is defined as the ratio of the number of received packets at the destination to the number of sent packets by the source. Since we use more and more relays, we have less

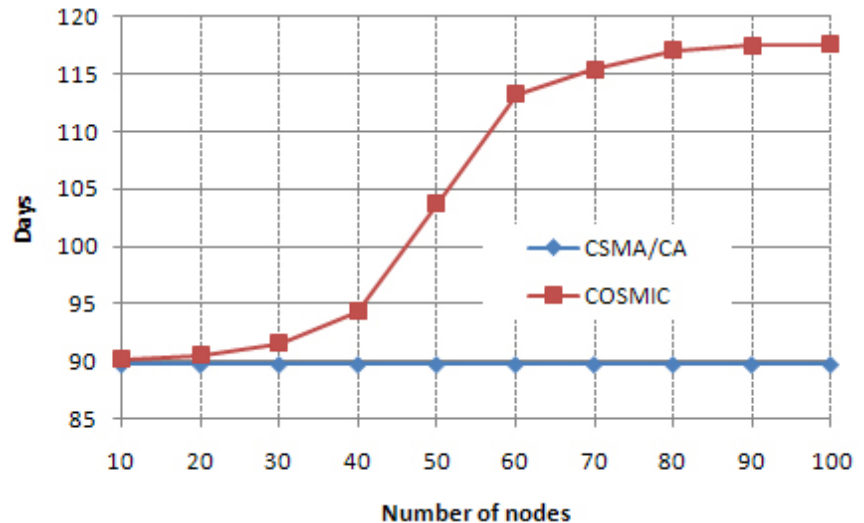

Fig. 6. Network Lifetime Vs Number of Nodes

packet loss. The delivery ratio is enhanced and reaches $95 \%$ when the node density reaches 100 nodes.

The outage ratio of the the first hop S-I (H1 in the figure)and the second hop I-D (H2 in the figure) are illustrated in Fig. 5. An outage happens when a packet is retransmitted by $\mathrm{S}$ or by I. The outage decreases as the node density increases and so the number of potential relays.

In the case of no cooperation, if an outage occurs the source re-transmits the packet until successful delivery or until attaining the maximal number of re-transmissions. But if we use cooperation with more nodes, we have greater chances to find a relay that have a better channel to the destination than the direct channel. In the case of 100 nodes, the outage ratio of the two hops become very low (around 2\%).

Fig .6 depicts the network lifetime defined as the time until the first node in the network dies. It increases from 90 days in communication without cooperation to reach around 118 days wih COSMIC (an increase of 25\%).

In fact, by adding more nodes to the network, we create more channels. Therefore additional nodes participate in the relaying of the packet to the next hop. This leads to a distribution of the energy consumption between the nodes and to a better use of the different channels of the network.

\section{CONClusion}

In this paper we proposed COSMIC; a cooperative MAC protocol for WSN with minimal control messages. In COSMIC relay selection is based on both the channel state information (CSI) and the remaining energy. We proved by simulation that using cooperative relaying increases the network lifetime by about $25 \%$ and the delivery ratio by 5 times. In addition, we proved that we can decrease the outage of the system. In future work we aim to enlarge the scope of our studies by implementing the protocol on a real testbed and by including Low Power Listening Mechanisms to reduce the activity of our nodes.

\section{REFERENCES}

[1] J. Laneman, D. Tse, and G. Wornell, "Cooperative diversity in wireless networks: Efficient protocols and outage behavior," Information Theory, IEEE Transactions on, vol. 50, no. 12, pp. 3062-3080, Dec. 2004. 
[2] N. Shastry, J. Bhatia, and R. Adve, "Theoretical analysis of cooperative diversity in wireless sensor networks," in Global Telecommunications Conference, 2005. GLOBECOM '05. IEEE, vol. 6, Dec. 2005, pp. 3269 3273.

[3] Z. Li and X.-G. Xia, "A simple alamouti space-time transmission scheme for asynchronous cooperative systems," Signal Processing Letters, IEEE, vol. 14, no. 11, pp. 804-807, 2007.

[4] H. Adam, C. Bettstetter, and S. M. Senouci, "Adaptive relay selection in cooperative wireless networks," in Personal, Indoor and Mobile Radio Communications, 2008. PIMRC 2008. IEEE 19th International Symposium on, 2008, pp. 1-5.

[5] A. Bletsas, H. Shin, and M. Win, "Outage-optimal cooperative communications with regenerative relays," in Information Sciences and Systems, 2006 40th Annual Conference on, March 2006, pp. 632-637.

[6] J. Zhang and Q. Zhang, "Cooperative routing in multi-source multidestination multi-hop wireless networks," in INFOCOM 2008. The 27th Conference on Computer Communications. IEEE, April 2008, pp. 2369 2377.

[7] Q. Cao, T. Abdelzaher, T. He, and R. Kravets, "Cluster-based forwarding for reliable end-to-end delivery in wireless sensor networks," in INFOCOM 2007. 26th IEEE International Conference on Computer Communications. IEEE, May 2007, pp. 1928-1936.

[8] P. Liu, Z. Tao, and S. Panwar, "A cooperative mac protocol for wireless local area networks," in Communications, 2005. ICC 2005. 2005 IEEE International Conference on, vol. 5, 2005, pp. 2962-2968 Vol. 5.

[9] B. Wang, Z. Han, and K. Liu, "Distributed relay selection and power control for multiuser cooperative communication networks using buyer/seller game," in INFOCOM 2007. 26th IEEE International Conference on Computer Communications. IEEE, May 2007, pp. 544 552.

[10] P. Q. Z. Z. Yan Chen, Guanding Yu, "Power-aware cooperative relay selection strategies in wireless ad hoc networks," Sept. 2006, pp. $1-5$.

[11] A. Nosratinia, T. Hunter, and A. Hedayat, "Cooperative communication in wireless networks," Communications Magazine, IEEE, vol. 42, no. 10, pp. 74-80, Oct. 2004.

[12] A. Bletsas, A. Khisti, D. Reed, and A. Lippman, "A simple cooperative diversity method based on network path selection," Selected Areas in Communications, IEEE Journal on, vol. 24, no. 3, pp. 659-672, March 2006.

[13] C.-T. Chou, J. Yang, and D. Wang, "Cooperative mac protocol with automatic relay selection in distributed wireless networks," in Pervasive Computing and Communications Workshops, 2007. PerCom Workshops '07. Fifth Annual IEEE International Conference on, March 2007, pp. 526-531.

[14] I. Rhee, A. Warrier, M. Aia, and J. Min, "Z-mac: a hybrid mac for wireless sensor networks," in SenSys '05: Proceedings of the 3rd international conference on Embedded networked sensor systems. New York, NY, USA: ACM Press, 2005, pp. 90-101. [Online]. Available: http://dx.doi.org/10.1145/1098918.1098929

[15] J. Polastre, J. Hill, and D. Culler, "Versatile low power media access for wireless sensor networks," in SenSys '04: Proceedings of the 2nd international conference on Embedded networked sensor systems. New York, NY, USA: ACM, 2004, pp. 95-107.

[16] S. H. Zanakis, A. Solomon, N. Wishart, and S. Dublish, "Multi-attribute decision making: A simulation comparison of select methods," European Journal of Operational Research, vol. 107, no. 3, pp. 507 - 529, 1998.

[17] www.xbow.com, "Micaz 2.4ghz," 2009 (accessed August 14, 2009). [Online]. Available: http://www.xbow.com/products/Product_pdf_files/ Wireless_pdf/MICA2_Datasheet.pdf 